23 | 1999

L'enseignement de la langue et de la littérature

françaises dans la seconde moitié du XIXe siècle

\title{
Sainte-Beuve, lu par Gustave Lanson et par Marcel Proust
}

Maria Hermínia Amado Laurel

\section{(2) OpenEdition \\ Journals}

Electronic version

URL: https://journals.openedition.org/dhfles/3051

DOI: 10.4000/dhfles.3051

ISSN: 2221-4038

Publisher

Société Internationale pour l'Histoire du Français Langue Étrangère ou Seconde

\section{Printed version}

Date of publication: 1 June 1999

Number of pages: p.271-291

ISSN: 0992-7654

\section{Electronic reference}

Maria Hermínia Amado Laurel, "Sainte-Beuve, lu par Gustave Lanson et par Marcel Proust", Documents pour l'histoire du français langue étrangère ou seconde [Online], 23 | 1999, Online since 14 September 2017, connection on 10 March 2023. URL: http://journals.openedition.org/dhfles/3051 ; DOI: https:// doi.org/10.4000/dhfles.3051

This text was automatically generated on 10 March 2023.

All rights reserved 


\title{
Sainte-Beuve, lu par Gustave Lanson et par Marcel Proust
}

\author{
Maria Hermínia Amado Laurel
}

1 À lire le titre de cette communication l'on pourrait s'interroger sur les raisons qui m'ont amenée à rapprocher trois personnages, Sainte-Beuve, Gustave Lanson et Marcel Proust, que rien, apparemment ne relie entre eux.

2 Le premier, " maître incontestable de la critique " (Lanson, G. : 1039) au long de plusieurs décennies; le second, auteur renommé de l'ouvrage d'histoire littéraire le plus répandu dans l'enseignement de la littérature française au Portugal jusqu'aux années 70, et Proust, finalement, le créateur de cette somme immense qui se soustrait au temps, A la recherche du temps perdu...

Deux raisons principales justifient pourtant mon choix.

D'une part, j'ai pu constater qu'à un certain moment de leur parcours intellectuel, Gustave Lanson et Marcel Proust avaient croisé ce personnage consacré par l'élite cultivée de son époque, et de la leur, mais qui n'en demeure pas moins ambiguë aujourd'hui, qu'a été Sainte-Beuve.

D'autre part, chacun de ces trois personnages a joué, et joue encore, peut-être, un rôle fondamental dans les études littéraires et dans l'enseignement de la littérature : SainteBeuve, dont les principaux textes s'établissent, grosso modo, entre 1829 et 1869, du côté de l'histoire de la critique, Lanson du côté de l'histoire littéraire, et Marcel Proust, dont la fiction romanesque est la mise en œuvre de toute la réflexion esthétique à laquelle il a voué sa vie, tout en constituant l'ouverture à la modernité littéraire au $\mathrm{XX}^{\mathrm{e}}$ siècle, espace privilégié où l'enseignement de la littérature se métamorphose en contemplation de la naissance de l'œuvre d'art.

Etant donné que la totalité de l'œuvre de Sainte-Beuve était achevée à la naissance des deux lecteurs dont je m'occuperai aujourd'hui - Lanson (1857-1920) avait à peine 12 ans lorsque Sainte-Beuve (1804-1869) est décédé, et Proust (1871-1922) ne verra le jour que trois ans après sa mort - il ne s'agira donc point ici d'analyser la réception de SainteBeuve du vivant de celui-ci, mais de réfléchir sur la contribution que deux 
personnalités aussi distinctes l'une de l'autre, mais proches dans le temps, ont pu donner à la construction de l'image de Sainte-Beuve. Cette image ne coïncide pas exactement, nous le verrons, avec celle qui a perduré, à de brefs intervalles, jusqu'au début des années 90 de notre siècle, en grande partie grâce au travail démolisseur de la " nouvelle critique " ${ }^{1}$ qui en a fait le maître des « curiosités » biographiques, pour ne pas dire le modèle d'un certain « voyeurisme » critique ${ }^{2}$.

7 L'écriture d'une histoire moderne de la littérature française ne pouvait passer sous silence l'importance de Sainte-Beuve, critique littéraire consacré déjà depuis bien longtemps.

8 Gustave Lanson a profondément réfléchi, en son temps, à la nécessité du renouvellement des études littéraires. Et ceci pour des raisons scientifiques, pédagogiques et socio-politiques qui s'imposaient.

9 Le panorama des études littéraires contemporain de Lanson était en effet dominé par un quatuor dont il s'efforça de montrer la dissonance : l'érudition, la critique, l'éloquence et la rhétorique. Aucune de ces quatre voix/ voies ne semblait pouvoir s'accorder à la discipline dominante (et dominatrice), dont l'orientation épistémologique s'étendait au travail sur les textes, fussent-ils de nature littéraire : l'Histoire.

10 Tombés dans le discrédit le plus complet, auquel a sans doute contribué le dogmatisme réactionnaire et désuet de Brunetière, lui-même discrédité dans les milieux universitaires de la Sorbonne et de l'Ecole normale au tournant du siècle, les études littéraires avaient besoin pour survivre d'une figure de référence et d'une méthode rassurante du point de vue scientifique. L'université se définissait alors selon la valeur des individus qui y détenaient les chaires, y exposaient leur savoir et la marquaient de l'empreinte de leur personnalité, plus que par un enseignement et des projets de recherche institutionnalisés, comme de nos jours.

Les études littéraires sont débordées sur le modèle de l'histoire, elles sont perdues, elles sont finies... ", constate Antoine Compagnon, en historien de l'université et du statut des littéraires et des études littéraires, au temps de Gustave Lanson (Compagnon, A. : 42).

11 Trouver une méthode de travail qui permette de mettre en lumière la "verité " littéraire fut un de ses tout premiers combats. Imbu de l'esprit scientifique de son siècle, tout en faisant preuve d'une intuition pédagogique remarquable portée sur la nécessité du renouvellement des études littéraires, perdues dans des " rhapsodies de Taine, Brunetière, Lemaître et Faguet »(Compagnon, A. : 57), Lanson cherche la méthode susceptible de respecter la singularité du fait littéraire et de concilier celle-ci avec le caractère historique et social des œuvres. En 1895, après avoir succédé l'année précédente à Brunetière à l'Ecole normale, il publie chez Hachette une œuvre qui ne connaîtra pas aussitôt le succès, ombragée par celle de Doumic, intitulée comme celleci, Histoire de la littérature française 3 , mais qui deviendra le dénominateur commun entre Lanson/Histoire littéraire/ Etudes littéraires/Enseignement de la littérature en peu de temps. Barthes le rappellera lorsqu'il associe l'acception courante de « littérature » à " ce qui s'enseigne $» . .$.

12 A partir de 1904, après son ascension à la Sorbonne et au poste de vice-président de la Société d'Histoire littéraire, la carrière de Lanson est tracée et celle de l'histoire littéraire également. 
13 En dressant le cadre de la critique et des études littéraires de son temps, Lanson n'hésite pas à considérer comme leur plus grave erreur une attitude que nous pouvons aisément constater de nos jours, bien que due à d'autres circonstances et motivations : l'écart du texte littéraire, l'absence de contact direct avec le texte, la non-lecture.

14 «La plupart des hommes [constatait déjà Sainte-Beuve] n'ont pas lu ceux qu'ils jugent » (cité par Antoine, G. : XLIII). Si, du temps de Lanson, cette faute impardonnable pouvait être imputée à Renan, qui avait écrit imprudemment dans son ouvrage, L'Avenir de la Science, la phrase transcrite par Lanson lui-même : «L'étude de l'Histoire littéraire est destinée à remplacer en grande partie la lecture directe des œuvres de l'esprit humain», de nos jours nous remarquons que c'est très souvent la lecture d'ouvrages critiques qui remplace celle des textes originaux, menant à ce que George Steiner a appelé pertinemment les lectures " tertiaires » (Steiner, G., 1993), pour ne pas faire référence à une autre attitude fréquente en milieu universitaire et qui consiste, chez les étudiants, à préférer la lecture d'éditions abrégées, telles que Profil d'une ouvre et d'autres semblables, constituant ce que j'appellerais des lectures nulles et non avenues.

15 Ayant développé sa pensée à l'occasion de nombreuses conférences et dans plusieurs articles, c'est peut-être dans deux textes préfaciels - les avant-propos à Histoire de la littérature française (1894) et à Hommes et Livres (1895) - et dans une conférence prononcée à l'université de Liège en 1904, que Lanson a le mieux exprimé son indépendance et plus tard sa dette envers Sainte-Beuve.

16 C'est à propos du besoin « d'érudition » dans les études littéraires que Lanson cite le nom de Sainte-Beuve : "L'étude de la littérature ne saurait se passer aujourd'hui d'érudition. [...] Mais il ne faut pas perdre de vue deux choses : l'histoire littéraire a pour objet la description des individualités [...] ; elle a pour base des intuitions individuelles ", affirme-t-il dans le premier des avant-propos cités (Lanson, G. : VII).

Chez Lanson, en effet, l'attrait exercé par la pensée positive ne l'a jamais écarté de la croyance profonde en la singularité et en l'individualité de l'œuvre littéraire. Si ses efforts pour systématiser toute la production littéraire française dans la somme fondamentale qu'est l'Histoire de la littérature française, reflètent de près l'esprit de recherche méthodologique rigoureuse qui caractérise la pensée scientifique de son époque, l'auteur a la conscience très nette des limites qu'offre à la connaissance du fait littéraire le transfert à ce domaine de méthodes qui, au départ, n'ont pas été conçues pour l'étude de la littérature : « Ni l'objet, ni les moyens de la connaissance littéraire ne sont, dans la rigueur du mot, scientifiques ", conclut-il dans le même texte (Lanson, G. : VIII).

18 Les " deux choses " soulignées par Lanson ainsi que cette dernière assertion nous ramènent immédiatement à Sainte-Beuve :

19 1. "L'histoire littéraire a pour base des intuitions individuelles », et nous pensons aux Portraits littéraires (série de cinquante textes inaugurée par La Revue des Deux Mondes en 1831) dont la conception esthétique élève l' intuition à la qualité majeure du critique.

20 Écoutons le critique dans son portrait de Diderot, où il compare la méthodologie de la composition d'un portrait littéraire aux étapes du travail du sculpteur sur pierre, qui, lui aussi «étudie», «retourne», «interroge à loisir» son matériau avant d'en faire une pièce vivante et conforme aux traits les plus saillants et les plus vrais de son modèle :

Chaque trait s'ajoute à son tour, et prend place de lui-même dans cette physionomie qu'on essaie de reproduire. [...] Au type vague, abstrait, général qu'une première 
vue avait embrassé, se mêle et s'incorpore par degrés une réalité individuelle, précise, de plus en plus accentuée et vivement scintillante ; on sent naître, on voit venir la ressemblance ; et le jour, le moment où l'on a saisi le tic familier, le sourire révélateur, la gerçure indéfinissable, la ride intime et douloureuse qui se cache en vain sous les cheveux déjà clairsemés, -à ce moment l'analyse disparaît dans la création, le portrait parle et vit, on a trouvé l'homme (Antoine, G. : 166).

La-lecture constitue la première étape de la méthode historique et littéraire proposée par Lanson. Cet auteur définit encore cette activité comme une affaire d'intuition et de goût. Et je reviens sur ses mots : «... la littérature n'est pas objet de savoir : elle est exercice, goût, plaisir. On ne la sait pas, on ne l'apprend, pas ; on la pratique, on la cultive, on l'aime ». Nous pourrions dresser la suite des correspondances implicites à ce projet :

« exercice » / lieu de travail ;

" goût » / fruit d'une sensibilité particulière, il fait appel et éveille notre propre sensibilité ; le goût se cultive, peut être objet de raffinement ;

« plaisir » / lieu de ravissement intellectuel et sensuel.

Chez Sainte-Beuve, le concept de lecture constitue également l'assise sur laquelle doit se fonder toute entreprise critique.

Dans sa Pensée XVIII, à la suite des Derniers Portraits littéraires, il distingue très clairement le rôle assigné au critique et les fonctions de la critique :

Je pense sur la critique deux choses qui semblent contradictoires et qui ne le sont

pas :

$1^{\circ}$ Le critique n'est qu'un homme qui sait lire et qui apprend à lire aux autres.

$2^{\circ}$ La critique, telle que je l'entends et telle que je voudrais la pratiquer, est une invention, une création perpétuelle (cité par Antoine, G. : XLII).

La lecture critique est donc définie, de même que chez Lanson, comme une affaire d'intuition, comme une activité profondément subjective, mise en parallèle avec l'acte créateur.

2. «L'histoire littéraire a pour objet la description des individualités ", et nous pensons à la connaissance de l'homme derrière ce qu'il écrit, derrière l'auteur, but auquel tendait la méthode sainte-beuvienne...

C'est à propos de ce point précis que Lanson veut se démarquer de la méthode de Sainte-Beuve. Dans une note de bas de page à son " Avant-propos " à l'Histoire de la littérature française, Lanson se défend de vouloir revenir « à la méthode de Sainte-Beuve et constituer une galerie de portraits".

Pourtant, dans son effort pour saisir dans la littérature ce qu'aucune méthode ne parvient à expliquer - « il reste souvent quelque chose que nulle de ces explications [le recours aux concepts tainiens de race, de milieu et de moment, et aux théories sur l'évolution des genres, introduites par Brunetière] n'atteint, que nulle de ces causes ne détermine : et c'est précisément dans ce résidu indéterminé, inexpliqué, qu'est l'originalité supérieure de l'œuvre... ", pressent Lanson... - se démarque-t-il vraiment de Sainte-Beuve, qui avait déjà alerté son lecteur, et dans un style bien plus imagé que celui de Lanson, envers " ce sceau unique de diamant " qui nous fait reconnaître les chefs-d'œuvre à travers les siècles, ces « perles » qu'il qualifie d'épithètes suggestifs : « limpides », " cristallines ", « radieuses » dans le portrait cité de Diderot ? (cité par Antoine, G. : 167). 


\section{à Hommes et Livres :}

Partir d'où Sainte-Beuve était parti, était excellent : mais il fallait n'en rester pas là.

Il avait donné une base solide aux études littéraires en ressuscitant l'individu, en donnant l'exemple de cette rare qualité : le sens de la vie.

Lanson a conscience de l'importance du travail de Sainte-Beuve, mais il estime qu'il faudra aller plus loin. Pour l'auteur de cette préface, l'individualité était à voir dans l'œuvre, dans chaque œuvre prise en elle-même, et non pas dans l'individu-auteur qui l'avait produite.

D'autre part, le regard que Lanson a porté sur l'éminent critique a connu aussi des changements de perspective au long de sa vie.

Confirmant ce qu'Antoine Compagnon démontre, dans sa fine analyse de l'idéologie non seulement littéraire mais aussi institutionnelle et politique des quatre grands maitres de la critique littéraire de la deuxième moitié du XIX siècle français, SainteBeuve (dont la carrière a cependant débuté pendant la première moitié du siècle), Taine, Brunetière et Lanson ${ }^{4}$, le Lanson des deux textes préfaciels cités, et le Proust de Contre Sainte-Beuve unissent leur voix pour réfuter la soumission de la critique saintebeuvienne au détail biographique. «Au lieu d'employer les biographies à expliquer les œuvres, [Sainte-Beuve] a employé les œuvres à constituer des biographies ", peut-on lire dans la préface à Hommes et Livres 5 .

Pourtant, Lanson semble se rapprocher de Sainte-Beuve, quelques années plus tard, lorsque, précisant sa méthode, il affirme la fonder sur la recherche inébranlable et poursuivie du « vrai », reprenant pour modèle l'auteur des Portraits, « un critique qui a eu le goût du vrai » et qu'il n'hésite pas à considérer, cette fois-ci, comme « le maître de la critique et le patron des critiques » dans une conférence produite à Liège en 1904 (Peyre, H. : 427--441).

Cette attitude me semble constituer un signe de l'émancipation idéologique et méthodologique du père de l'histoire littéraire par rapport à la maitrise intellectuelle exercée à l'époque par Taine et par Brunetière ${ }^{6}$. Cette maîtrise est fortement implicite dans la conférence citée.

D'autre part, elle me semble constituer un des premiers moments de la réhabilitation de Sainte-Beuve, laquelle ne sera reprise avec entrain que bien des années après la mort du critique, ainsi que je l'ai constaté au début de ce travail. Écoutons Lanson en 1904 : " Je me hâte de dire que Sainte--Beuve, s'il a eu ses faiblesses, ses tares, vaut mieux, tout de même, que sa réputation » (Peyre, H. : 428). Réputation, il va sans dire, que Lanson avait lui-même contribué, quelques années auparavant, à répandre...

-L'effort de réhabilitation de Sainte-Beuve entrepris par Lanson mettra en valeur :

-son goût pour le « vrai », objectif à atteindre et qui n'est pas, forcément, synonyme d'« objectivité » ni d'« impartialité », mais plutôt de la liberté qui doit caractériser l'esprit critique ;

-sa « profonde intelligence de toutes les inquiétudes humaines";

-son désir de ne pas préférer le beau au vrai, ni de prendre le beau pour le vrai ;

-sa sensibilité « aux aspects esthétiques » qui en a fait un " poète, artiste en vers, disciple attentif des nouveautés métriques du romantisme »; 
43 notamment en ce qui -concerne son incapacité à discerner les valeurs de la littérature contemporaine : «Assurément, Sainte-Beuve a ses limites, ses partis pris. Il commet des erreurs, des injustices. Il a eu longtemps un préjugé politique. Il a un fond de goût classique que son romantisme n'a fait que recouvrir un moment, qui ne se laisse pas entraîner au naturalisme et aux coups de vigueur un peu brutale de la littérature de Second Empire » (Peyre, H. : 438). nonobstant le moment de la carrière de Lanson auquel elle correspond, et ses intentions critiques face au poids de l'orientation des études littéraires selon l'empire de Brunetière et du tout puissant Hippolyte Taine ${ }^{7}$, renchérit sur les aspects positifs identifiés par Lanson.

53 C'est encore sur les dons stylistiques de son écriture, sur la sensibilité de l'auteur à certains sujets et personnalités littéraires, sur son « art » du portrait, sur sa défense de la liberté créatrice pour l'activité critique, aussi bien que sur le prix qu'il accorde à l'indépendance du critique face aux systèmes normatifs, que la critique saintebeuvienne de nos jours revient, pour les mettre en valeur.

54 Lisons-le, dans un passage des Causeries du Lundi :

... variez sans cesse vos études, cultivez en tous sens votre intelligence, ne la cantonnez ni dans un parti, ni dans une école, ni dans une seule idée ; ouvrez-lui 
des jours sur tous les horizons ; portez-vous avec une sorte d'inquiétude amicale et généreuse vers tout ce qui est moins connu, vers tout ce qui mérite de l'être, et consacrez-y une curiosité exacte et en même temps toujours émue ;

- ayez de la conscience et du sérieux en tout ; évitez la vanterie et jusqu'à l'ombre du charlatanisme (...);- voyez la société et ce qu'on appelle le monde pour en faire profiter les Lettres ; cultivez les Lettres en vue du monde, et en tâchant de leur donner le tour et l'agrément sans lesquels elles ne vivent pas ; (...) et si vous ne dites pas tout le vrai, n'écrivez jamais le faux... (cité par Antoine, G. : III).

Nous assistons aujourd'hui à l'exorcisme du "démon de la théorie ", à un renouveau de l'histoire littéraire, à un retour à la biographie, et la critique s'intéresse à nouveau au journal d'écrivain, à l'écriture en marge, au paratexte.

N'est-ce pas l'occasion de nous remettre à la lecture sans parti pris des Portraits? Ne faudrait-il pas réévaluer sa méthode?

La contestation à laquelle Proust soumet la méthode critique de Sainte-Beuve coïncide avec les débuts de sa carrière d'écrivain ${ }^{9}$, et se révèle être ainsi une occasion précieuse qui s'offre à lui de réfléchir sur ses propres choix esthétiques ${ }^{10}$.

Il me semble que j'aurais ainsi à dire sur Sainte-Beuve, et bientôt beaucoup plus à propos de lui que sur lui-même, des choses qui ont peut-être leur importance, qu'en montrant en quoi il a péché, à mon avis, comme écrivain et comme critique, j'arriverais peut-être à dire, sur ce que doit être la critique et sur ce qu'est l'art, quelques choses auxquelles j'ai souvent pensé (Proust, M. : 219),

considère Marcel Proust au tout début de son texte sur « La méthode de Sainte-Beuve $»^{11}$

Son projet de réflexion semble vouloir être d'autant plus novateur ${ }^{12}$ (et provocateur) qu'il est conçu et développé dans les années qui suivent les commémorations du centenaire de la naissance de Sainte-Beuve en 1804, ainsi que l'a bien remarqué Pierre Clarac dans la Notice à Contre Sainte-Beuve de l'édition de La Pléiade (Proust, M. : 819 (1)). Plus précisément, les différents fragments qui le composent ont été produits entre 1905 et 1909, date à partir de laquelle Proust se voue entièrement à l'écriture de la Recherche.

Du point du vue de l'histoire de la critique, la position de Proust contre le biographisme historiciste (la vieuvre dont parlait Antoine Compagnon dans La Troisième République des lettres, depuis lors identifiée à la démarche méthodologique exclusive de l'histoire littéraire) s'insère dans le mouvement de contestation auquel donneront leur voix des penseurs contemporains tels que B. Croce, Ch. Péguy, T. S. Eliot, Valéry, Gide, entre autres ou, plus tard, Malraux dans Les Voix du silence.

61 La réflexion de Proust sur la méthode biographique de Sainte-Beuve s'insère dans le cadre des préoccupations esthétiques et littéraires qui l'ont accompagné pendant toute sa vie. «La présence de l'artiste dans ce qu'il fait » renvoie, dans le cas de Proust, au problème majeur de la réflexion esthétique générale : celui des rapports entre la réalité et l'art. Cette question s'est manifestée dès ses premiers écrits, et elle est déjà bien explicite dans Jean Santeuil : " quels sont les rapports secrets, les métamorphoses nécessaires qui existent entre la vie d'un écrivain et son œuvre, entre la réalité et l'art, ou plutôt, comme nous pensions alors, entre les apparences de la vie et la réalité même qui en faisait un fonds durable et que l'art a dégagé », constituent des interrogations de deux personnages de cet ouvrage inachevé. représenterait à son tour tout l'itinéraire d'une recherche autour de la question des 
rapports entre l'expérience biographique et la création artistique, dans ce cas, narrative. Cette recherche, d'abord exercée sur ce que le narrateur peut vérifier à propos des artistes dont il prise l'œuvre, mais ayant eu des résultats décevants (rappelons-nous la rencontre de Bergotte, par exemple), est vécue, à la fin du livre, dans son cas personnel. Son livre est, en réalité, l'histoire de la prise de conscience de soi-même en tant que narrateur de son œuvre, en tant qu'écrivain. Ce n'est pas à d'autres qu'il laisse le soin de la raconter mais il s'en fait le narrateur-même, sans quoi l'œuvre n'existerait pas. Lui seul, premier narrateur et premier lecteur est aussi, et en rigueur, son seul destinataire. CEuvre fermée avant toutes, que la Recherche...

Je voudrais m'arrêter uniquement sur ce qui me semble être le point de départ de la contestation de la méthode critique de Sainte-Beuve par Marcel Proust : précisément la question de la représentation de l'écrivain dans son œuvre. connaissance : de l'auteur pour le premier, du lecteur pour le second. « La lecture est pour nous l'incitatrice dont les clefs magiques nous ouvrent au fond de nous-mêmes la porte des demeures où nous n'aurions pas su pénétrer » soutient Marcel Proust dans ses Journées de lecture (Proust, M. : 180).

6 Paradoxalement pourtant, ces deux conceptions présupposent, chez les deux auteurs, une démarche doublement coïncidente de la part du lecteur : la fusion du critique, en tant que lecteur, dans l'objet de sa lecture, d'une part, et, d'autre part, une même attitude devant le texte: la lecture comme dépassement du texte à lire. Chercher l'auteur derrière le texte, pour Sainte-Beuve, se découvrir soi-même, pour Proust. L'ultime conséquence de cette fusion donnera lieu à l'expérience du pastiche chez les deux écrivains ${ }^{13}$

67 Sainte-Beuve désigne cette fusion par l'expression de "métamorphose »: «je tâche de disparaître dans le personnage que je reproduis, je me fais à lui, même par le style, j'emprunte et je revêts sa diction ", affirme-t-il dans une page du Cahier vert (cité par Antoine, G. : LII) ou bien: "Je suis l'esprit le plus brisé et le plus rompu aux métamorphoses » (cité par Dotoli, G : 209).

68 Pour Proust, le lecteur, à force de lire profondément, retrouve dans les livres des vérités sur lui-même auxquelles il ne serait pas parvenu en de-hors de la lecture ; la lecture devient ainsi pouf lui le moment de l'« écriture ", de la vérité profonde de soimême, donc, le moment de la métamorphose du lecteur en écrivain (comme dans la Recherche, où le lecteur se révèle l'auteur matériel du récit) et en auteur, puisque créateur, maître d'une autre partie de soi-même, des vérités cachées, en lui-même et qui lui deviennent reconnaissables par cet acte ( $d$ 'où la subjectivité inhérente à tout acte de lecture, son caractère unique et non partageable).

9 C'est surtout à l'auteur des Causeries que Marcel Proust s'en prend le plus particulièrement, peut-être dans le but de se démarquer d'un critique trop " encombrant ", dont l'ombre planait encore sur l'opinion critique de son temps, la preuve en est dans les commémorations du centenaire en $1904^{14}$.

70 La conception de la lecture chez Marcel Proust, très clairement exposée dans le texte Journées de lecture, s'oppose à la notion de lecture comme conversation avec les « bons livres ", partagée par Lanson et par Sainte-Beuve. Ce mot de Descartes est transcrit dans l'Avant-propos à l'Histoire de la littérature française, et par Proust lui-même qui fait 
le résumé de la thèse soutenue par Ruskin dans Sésame et les lys dont il a entrepris la traduction en $1904^{15}$ : " la lecture des bons livres est comme une conversation qu'on aurait avec les plus honnêtes gens des siècles passés, et une conversation où ils ne nous livreraient que le meilleur de leurs pensées » (Lanson, G. : VIII ; Proust, M. : 173).

Dans son commentaire à l'œuvre de Ruskin, Sésame et les lys, Proust considère que la " conversation » « dissipe immédiatement » le " travail fécond de l'esprit sur lui-même » stimulé par la lecture solitaire (Proust, M. : 174).

Le hors-texte auquel renvoie le concept de lecture de Marcel Proust l'écarté définitivement des notions de lecture-conversation, et de conversation-source de connaissance d'autrui défendues par Sainte-Beuve, et s'affirme en tant que chronotope par excellence de la lecture.

En effet, pour l'auteur de Journées de lecture, c'est au moment de l'interruption ou de l'arrêt de la lecture que la vraie lecture commence. Ainsi, à l'expression employée par les écrivains en fin de volume, "Conclusions ", et qui correspond normalement au dernier chapitre, Proust préférerait employer celle d'« Incitations ». Et je me permettrais de rappeler son sentiment à ce sujet, exprimé dans un passage de Journées de lecture : "La lecture est pour nous l'incitatrice dont les clefs magiques nous ouvrent au fond de nous-mêmes la porte des demeures où nous n'aurions pas su pénétrer» (Proust, M. : 180).

74 "La lecture " pour lui « est au seuil de la vie spirituelle; elle peut nous y introduire : elle ne la constitue pas ». En effet, chez Proust, l'acte de lecture en lui-même, la durée de la lecture, sont éphémères : tout ce qu'ils peuvent éveiller en nous ce sont " des désirs ", pas des réponses définitives. Celles-là, c'est en nous-mêmes qu'il nous faudra apprendre à les " voir » (Proust, M. : 176-178). Regarder, apprendre à voir, ce sont ses leçons de lecture, inspirées par les vers de son amie Anna de Noailles qu'il transcrit dans Journées de lecture ${ }^{16}$ : « Regarde la maison de Zélande, rose et luisante comme un coquillage : Regarde! Apprends à voir ! (Proust, M. : 178).

Pour Proust, les livres sont tout son univers : extérieur et intérieur. Chez lui, conclura Luc Fraisse, dans son étude de l'idéologie esthétique de l'auteur publiée en 1995, " chaque lecteur est quand il lit le propre lecteur de soi-même » (Fraisse, L. : 172). La lecture-critique de Sainte-Beuve aboutit naturellement à l'inventaire des biographies d'auteur ; la lecture de Proust à la connaissance de soi. Toutes deux dans un hors-texte portant sur des objets distincts : l'auteur pour l'un, le lecteur pour l'autre.

Si ces deux itinéraires de lecture prétendent aboutir à la connaissance d'objets distincts, les signes qui les jalonnent sont également de nature différente.

Selon Proust, la méthode de Sainte-Beuve, parce qu'elle ne s'attache qu'aux aspects extérieurs, observables, localisables, identifiables, dans un souci d'exhaustivité tout superficiel, est insensible à la complexité de l'acte créateur, elle « méconnaît ce qu'une fréquentation un peu profonde avec nous-mêmes nous apprend : qu'un livre est le produit d'un autre moi que celui que nous manifestons dans nos habitudes, dans la société, dans nos vices. Ce moi-là, si nous voulons essayer de le comprendre, c'est au fond de nous-même, en essayant de le recréer en nous, que nous pouvons y parvenir.

[...] Cette vérité [poursuit Marcel Proust], il nous faut la faire de toutes pièces. [...] Il est trop facile de croire qu'elle nous arrivera un beau matin dans notre courrier, sous forme d'une lettre inédite qu'un bibliothécaire de nos amis nous 
communiquera, ou que nous la recueillerons de la bouche de quelqu'un qui a beaucoup connu l'auteur ". sur les données immédiates de la conscience avait paru en 1889, ne doivent pas être confondues par la critique, ainsi que l'a fait Sainte-Beuve, qu'il n'hésite pas à accuser de ne pas « avoir vu l'abîme qui sépare l'écrivain de l'homme du monde, pour n'avoir pas compris que le moi de l'écrivain ne se montre que dans ses livres, et qu'il ne montre aux hommes du monde [...] qu'un homme du monde comme eux ».

81 L'attrait exercé par le moi social de quelques écrivains auprès du public des salons, plus ou moins sensible à leur « esprit ", fait méconnaître à Sainte-Beuve, selon Proust, trop d'écrivains contemporains de valeur (tels Chateaubriand, Baudelaire, Vigny, Stendhal, Mérimée) pour que sa méthode puisse être reconnue valable, justifiant ainsi sa position contre Sainte-Beuve. D'autre part, cette méthode, en grande partie nourrie de l'opinion que les gens ayant connu l'écrivain dont il s'agit de tracer le portrait ont pu exprimer, nourrie de témoignages pour la plupart indirects, génère " une œuvre écrite avec l'inconsciente collaboration des autres [et, en conséquence, en devient-elle] moins personnelle» (Proust, M. : 228) ${ }^{18}$.

ette opinion est à compléter par le refus proustien de la mémoire "volontaire " à laquelle fait constamment appel la reconstruction du passé par Sainte-Beuve, au profit de la mémoire " involontaire » qui caractérise le phénomène du souvenir chez Marcel Proust.

ignes identificateurs de l'artiste dans le monde réel se révèlent en réalité bien illusoires au narrateur de la Recherche curieux de rencontrer ces personnages qu'il avait mythifiés à la rencontre de leur œuvre. En effet, aucune ligne de continuité ne semble s'établir entre l'image qu'il se faisait de l'être qui avait pu produire de pareils chefs-d'œuvre et les êtres/ personnages de Bergotte, Vinteuil, Elstir ou la Berma qui se présentent devant ses yeux. Pourtant son admiration pour leur œuvre n'en sort pas diminuée.

La conversation s'opposerait chez Proust au secret, à l'indicible dont s'enveloppe le moi intime de l'artiste, ce qui nous permet de mieux comprendre son refus du dilettantisme dans l'art, de même que les excès d'érudition et de futilité mondaines dont il accuse Sainte-Beuve.

L'opposition est nette entre des personnages de la Recherche tels que Saint-Loup, l'amateur d'art, et Octave, le vrai artiste pour qui

les choses de l'art devaient être quelque chose de si intime, de vivant tellement dans les plus secrets replis de lui-même qu'il n'eût sans doute pas eu l'idée d'en parler comme eût fait Saint-Loup par exemple, pour qui les arts avaient le prestige que les attelages avaient pour Octave (Fraisse, L. : 28).

Ainsi se dessine ce que j'appellerais l'esthétique de l'absence chez Marcel Proust et qui trouve ses exemples les plus explicites et les plus connus dans des passages comme celui de la petite madeleine ou des pavés de Venise, dont les critiques se servent habituellement pour illustrer les manifestations de la fameuse « mémoire involontaire ».

Documents pour l'histoire du français langue étrangère ou seconde, 23 | 1999 
C'est d'ailleurs cette esthétique qui informe la conception globale de la Recherche.

Dans cet esprit, l'artiste est celui qui mène une double existence, qui partage l'univers social contemporain (ou mieux, dans le cas concret des héros de Proust, des milieux de choix dans la société), tout en existant véritablement en dehors de ces milieux, dans l'intérieur de soi-même. C'est là qu'il organise son existence, c'est là que par des coïncidences inexplicables d'un point de vue rationnel - tel que le soutenait la « conception intellectualiste de la réalité » de Taine -, le vrai sens des mots et des événements vécus se révèle à lui. L'artiste est ainsi chez Proust un être bien en avance sur ses contemporains, sur ses proches, puisqu'il accède à une connaissance de la réalité intérieure qui leur échappe, trop occupés qu'ils sont de l'apparence des choses (à la manière de Sainte-Beuve ${ }^{19}$.

9 En concluant, Lanson prise le maître dans un effort de libération, d'autonomie pour l'affirmation d'une méthode qu'il voulait strictement littéraire, c'est-à-dire indépendante des méthodes des autres sciences, capable de mettre en évidence ce « reste " qu'est la littérature, quand on a tout dit sur la race, le milieu et le moment, et aussi pour se faire une place dans un univers critique dominé par le dogmatisme ou l'impressionnisme superficiel ; Proust médit de lui parce que ce reste, ces perles, ce n'est pas dans la conversation qu'on les trouve, la conversation ne pouvant nous aider qu'à tracer de l'extérieur le parcours existentiel de l'auteur, mais dans la séparation nette entre l'homme social et l'homme intime qui existent dans chacun de nous.

Lanson dans le champ méthodologique (recherche d'une méthode exclusive et exhaustive pour les études littéraires) et institutionnel (question d'affirmation personnelle, institutionnelle et politique) ; Proust dans le champ de la création littéraire, recherchant la nature du phénomène littéraire, manifestée dans ses rapports avec le créateur et le réel, aucun des deux n'a pu effacer l'ombre de Sainte-Beuve.

\section{BIBLIOGRAPHY}

ANTOINE, Gérald (1993), Sainte-Beuve : Portraits littéraires, Paris, Robert Laffont.

COMPAGNON, Antoine (1983), La Troisième République des lettres : de Flaubert à Proust, Paris, Seuil.

DoToLI, Giovanni (1989), « Sainte-Beuve, critico moderno », Bérénice, anno XI, 27 : 207-229.

FRAISSE, Luc (1995), L'Esthétique de Marcel Proust, Paris, Sedes.

LANSON, Gustave (1970), Histoire de la littérature française, Paris, Hachette, (I édit. 1892).

ORLANDO, Francesco (1970), « Proust, Sainte-Beuve e la ricerca in direzione sbagliata », Critica e storia letteraria, II : 226-250.

PEYRE, Henri (1965), Gustave Lanson : Essais de méthode, de critique et d'histoire littéraire, Paris, Hachette.

PROUST, Marcel (1971), « Projets de préface », Contre Sainte-Beuve, précédé de Pastiches et mélanges et suivi de Essais et articles, Paris, Gallimard/Pléiade : 211-218.

Documents pour l'histoire du français langue étrangère ou seconde, 23 | 1999 
PRoust, Marcel (1971), «La méthode de Sainte-Beuve», Contre Sainte-Beuve, précédé de Pastiches et mélanges et suivi de Essais et articles, Paris, Gallimard/Pléiade : 219-232.

STEINER, George (1993), Presenças reais, Lisboa, Edições Presença.

\section{NOTES}

1. Remarquons que la "nouvelle critique " utilisera les mêmes arguments que Lanson avait déjà employés contre la critique " traditionnelle », en particulier ceux de " dogmatisme » et d'« impressionnisme », lorsqu'elle s'attaquera à l'héritage de Sainte-Beuve et au " 1 an son i s me ».

2. Je citerai quelques lecteurs contemporains avertis de Sainte-Beuve : Jean-Pierre Richard, Etudes sur le Romantisme, 1970 ; Giovanni Dotoli, « Sainte-Beuve, critico moderno », Bérénice, anno XI, n 27, 1989 : 207-229 et, plus récemment, le redressement de Sainte-Beuve entrepris par Michel Balzamo qui a publié une anthologie critique aux Editions Universitaires en 1990, par Bruno de Cessole dans un article sur la réhabilitation du critique paru dans le Figaro littéraire du 29 juin 1992, et par Gérald Antoine dont l'édition critique des Portraits littéraires est parue en 1993.

3. Nonobstant cette coïncidence, et sans que je puisse préciser si elle a été voulue par Lanson, dans un dessein provocatoire à ce qui constituait " la tradition ", les deux ouvrages renferment deux conceptions opposées sur l'histoire littéraire, celle de Doumic représentant « l'envers même de la future histoire littéraire », comme le constate Antoine Compagnon (1983 : 44).

4. Compagnon, Antoine, La Troisième République des lettres : de Flaubert à Proust, 1983 : 174-181.

5. Consultable à la Bibliothèque nationale en microfiche, et inséré par Henri Peyre dans le recueil d'articles et de conférences de Lanson publié sous le titre : Essais de méthode, de critique et d'histoire littéraire, Paris, Hachette, 1965 : 443- 450.

6. Voir l'analyse de ce renversement d'opinion interprété comme manœuvre stratégique de libération personnelle de cette maîtrise in Compagnon, Antoine, op. cit. : 175.

7. La position de Lanson, contre les « positions dogmatiques ", l'« esprit de système ", la formulation d'une " hypothèse générale » qui guide et limite l'explication, l'apport " dans un portrait individuel [d']une explication de l'ensemble des choses ", la construction d'une « philosophie et [d']une esthétique à propos d'un romancier ou d'un poète » semble renvoyer clairement à l'affirmation de son opposition à la critique « scientifique " appliquée aux études littéraires, qui ne soit pas strictement littéraire. Son retour à Sainte-Beuve a peut-être constitué un stratagème, comme le suggère Antoine Compagnon ; n'empêche qu'il met en évidence la valeur du travail de Sainte-Beuve, tout comme le scepticisme fin-de-siècle dont témoigne le dernier Lanson : "Gardons-nous d'être trop simpliste dans le choix d'une méthode pour nés études littéraires ».

8. Cf. le dernier titre publié par Antoine Compagnon, Seuil, 1998.

9. L'opposition à Sainte-Beuve semble avoir occupé l'esprit de Marcel Proust assez tôt dans son parcours d'écrivain, au moins depuis l'article «Sur la lecture », publié en 1905 dans La Renaissance latine, article qui devrait être apposé comme préface à la traduction de l'ouvrage de J. Ruskin, Sésame et les lys (achevée d'imprimer en 1906), et qui prendrait le titre Journées de lecture (Proust, M., 1971 : 788-789) lors de son insertion dans Pastiches et Mélanges (recueil qui comprend des textes produits entre 1900 et 1908, publiés en volume en 1919) (Proust, M., 1971 : 682).

10. Sainte-Beuve, texte resté incomplet et à l'état d'essai littéraire, avait au départ été conçu par Marcel Proust comme un projet de plus longue haleine, une « nouvelle » peut-être (lettre du 16 mai 1908 à Robert Dreyfus, citée par Pierre Clarac dans la Notice à Contre Sainte-Beuve, Pléiade : 821). Ce projet sera abandonné à partir de 1909, date après laquelle Proust se consacre entièrement à l'écriture de la Recherche. Pourtant des personnages et des situations d'abord 
conçus pour ce texte réapparaîtront dans la Recherche, confirmant ainsi l'unité globale des écrits proustiens.

11. Outre l'ensemble des textes qui constituent le Contre Sainte-Beuve, il faudrait citer également Journées de lecture et le pastiche intitulé «Critique du roman de M. Gustave Flaubert sur l'Affaire Lemoine " par Sainte-Beuve, dans son feuilleton du Constitutionnel (Proust, M., 1971 : 16-21).

12. Par rapport à la tradition romantique, positiviste ou naturaliste (Orlando, $R, 1970: 229$ ).

13. Plus particulièrement chez Proust, l'écriture de pastiches a constitué une étape préparatoire à la fusion totale qu'est la Recherche.

14. L'étude des rapprochements possibles entre Proust et Sainte-Beuve reste pourtant encore à faire. L'esthétique de Marcel Proust semble se laisser pénétrer par celle de Sainté-Beuve sur quelques points précis, notamment : l'écriture à la première personne;-le récit de l'expérience personnelle du narrateur ;-le mixage stylistique de l'essai « La méthode de Sainte-Beuve », où la scène racontée semble avoir été vécue par le narrateur lui-même.

15. La traduction de Ruskin a offert l'occasion à Proust d'exposer ses "idées ", tout en les opposant à celles du critique anglais. La préface à sa traduction constitue ainsi « une sorte de critique indirecte de sa doctrine » (Proust, M., 1971: 793).

16. Cf. Proust, M. : 802, note 2 à la page 176 .

17. Jean Rousset, Forme et signification. Essais sur les structures littéraires de Corneille à Claudel Paris, Corti, $1964: 150$.

18. C'est pourquoi les jeunes artistes qui voulaient se faire une place dans l'opinion publique de leur temps aspiraient à un Lundi de Sainte-Beuve, à un article dans un journal signé de lui (cf. l'importance de la notice de Sainte-Beuve à l'édition originale de Gaspard de la Nuit d'Aloysius Bertrand, en 1842, d'après le portrait qu'il avait publié de ce romantique incompris dans le numéro de La Revue de Paris du 24 juillet 1842, et les raisons occultes pour lesquelles Baudelaire a peut-être pris cet auteur pour modèle de référence de ses Petits poèmes en prose : parce qu'il avait été préfacé par Sainte-Beuve, un des critiques les plus cotés de l'époque).

19. Selon Luc Fraisse « cette doctrine fut enseignée à Proust à la Sorbonne en 1894-95 » par Gabriel Séailles, son professeur d'esthétique (Fraisse, L. : 29-30).

\section{AUTHOR}

\section{MARIA HERMÍNIA AMADO LAUREL}

Universidade de Aveiro 